\title{
Spin-valley Hall phenomena driven by Van Hove singularities in blistered graphene
}

\author{
M. Umar Farooq $\mathbb{D}^{1,5}$, Arqum Hashmi $\mathbb{D}^{2,3,5 凶}$, Tomoya Ono ${ }^{4}$ and Li Huang $\mathbb{D}^{1 凶}$
}

Using first-principles calculations, we investigate the possibility of realizing valley Hall effects (VHE) in blistered graphene sheets. We show that the Van Hove singularities (VHS) induced by structural deformations can give rise to interesting spin-valley Hall phenomena. The broken degeneracy of spin degree of freedom results in spin-filtered VH states and the valley conductivity have a Hall plateau of $\pm \mathrm{e}^{2} / 2 h$, while the blistered structures with time-reversal symmetry show the VHE with the opposite sign of $\sigma_{x y}^{K / K^{\prime}}\left(\mathrm{e}^{2} / 2 h\right)$ in the two valleys. Remarkably, these results show that the distinguishable chiral valley pseudospin state can occur even in the presence of VHS induced spin splitting. The robust chiral spin-momentum textures in both massless and massive Dirac cones of the blistered systems indicate significant suppression of carrier back-scattering. Our study provides a different approach to realize spin-filtered and spin-valley contrasting Hall effects in graphene-based devices without any external field.

npj Computational Materials (2020)6:197; https://doi.org/10.1038/s41524-020-00470-9

\section{INTRODUCTION}

The quantum anomalous Hall effect (QAHE) in 2D materials arises from the complex interplay between time-reversal symmetry breaking and spin-orbit coupling (SOC). In contrast with the quantum Hall effect (QHE), which can be achieved via a strong external magnetic field, QAHE is solely associated with eternal magnetization and SOC of materials ${ }^{1,2}$. The most common way to achieve QAHE is through the induction of exchange splitting in topological insulators ${ }^{3-6}$. Thus, the quantization of Hall conductance is correlated with the topological number, which is robust against minor disorder and perturbations ${ }^{7}$. Furthermore, the interplay of the SOC and broken spatial inversion gives rise to valley degrees of freedom for Bloch electrons $s^{8,9}$. The valley is represented by a binary pseudospin in reciprocal space, and it usually occurs at the edges of the hexagonal Brillion zone (BZ) ${ }^{10,11}$. The prospect of being able to manipulate the valley pseudospin in a topologically nontrivial material shows great promise for nextgeneration electronic devices ${ }^{12}$. The valley binary degree of freedom is conceptually equivalent to the spin degree of freedom for a single electron. However, the lack of associated physical quantities for the valley pseudospin makes it challenging for external manipulation.

Graphene has been the focus of intense research in recent years, mainly because of its unusual properties, such as the remarkable feature of Dirac fermions ${ }^{13,14}$. The long electron spin relaxation time in graphene makes it a strong candidate for spintronics and valleytronics applications. However, the bipartite honeycomb structure with equivalent $A$ and $B$ sublattice enforces the Berry curvature of $\mathrm{K}$ into $-\mathrm{K}$ and renders them indistinguishable. In this regard, strain engineering has been pursued intensively to break this degeneracy and modify the electronic properties ${ }^{13,15,16}$. Previous reports have shown that strain in graphene induces a shift in the low-energy Dirac spectrum because of the spatial change in the electron density and results in an effective vector potential between the two sublattices ${ }^{17}$. This strain-induced gauge field can give rise to large pseudo-magnetic fields (PMF), which have opposite signs in the $K$ and $K^{\prime}$ valleys ${ }^{18-21}$. Therefore, this gauge field can be used as a building block for the valleytronics device.

Several methods have been used to produce controllable strain fields, such as the direct application of pressure through STM tips $^{21,22}$, dislocations or grain boundaries ${ }^{23,24}$, gas inflation ${ }^{25}$, and electron radiation ${ }^{26,27}$. These experimental methods create nanobubbles and blisters in graphene sheets ${ }^{28}$. The simplest form of these structures are clusters of Stone-Wales defects, where the rotated carbon-carbon bonds create adjacent pentagonal and heptagonal rings ${ }^{23,24,29}$. Such blisters exhibit out-of-plane deformation of different sizes and shapes. The PMF associated with these nano blisters varies spatially and is usually of a large magnitude. In addition, they also create flat bands and Van Hove singularities (VHS), which open up the possibility of magnetic states in graphene ${ }^{30}$.

In this study, we focus on graphene blisters, which have been created via electron beam irradiation in a previous report ${ }^{31}$. Using first-principles calculations, we investigate the possibility of realizing valley Hall effects (VHE) in graphene. We show that the strain field due to local deformation of the blisters results in a lowenergy VHS around the Fermi level and induces a magnetic state in graphene. Recently, it has been demonstrated that the coexistence of PMF and magnetic fields allows for effective control of the valley in graphene ${ }^{32}$. Here, we show that the interplay between PMF and VHS gives rise to spin-filtered $\mathrm{VH}$ states. Interestingly, the chirality of the valley pseudospin state is preserved even with a magnetic state. Besides that, the spinor wave functions obtained from non-collinear calculations show strong chiral spin-momentum textures in all blisters, which suggests significant suppression of carrier back-scattering. This finding provides an opportunity to realize spin and valley contrasting Hall effects in graphene for valleytronics and spintronics device applications.

${ }^{1}$ Department of Physics, Southern University of Science and Technology, Shenzhen, Guangdong 518055, China. ${ }^{2}$ Center for Computational Sciences, University of Tsukuba, Tsukuba, Ibaraki 305-8577, Japan. ${ }^{3}$ Kansai Photon Science Institute, National Institutes for Quantum and Radiological Science and Technology (QST), Kyoto 619-0215, Japan. ${ }^{4}$ Department of Electrical and Electronic Engineering, Kobe University, Kobe 657-8501, Japan. ${ }^{5}$ These authors contributed equally: M. Umar Farooq, Arqum Hashmi.

花mail: hashmi.arqum@qst.go.jp; huangl@sustech.edu.cn 


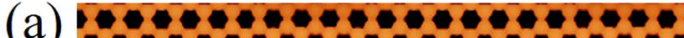

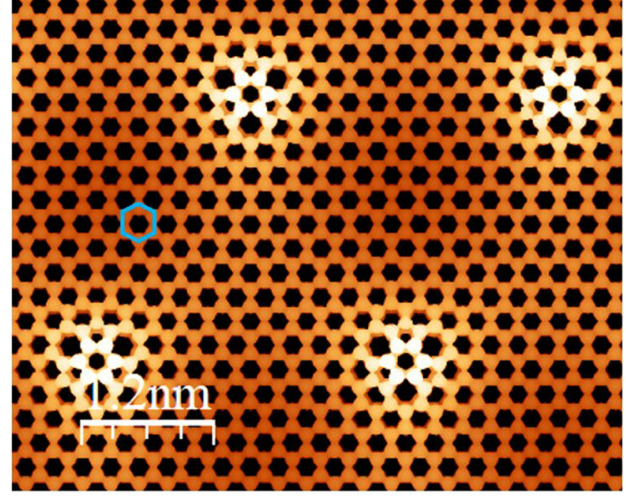

(b)

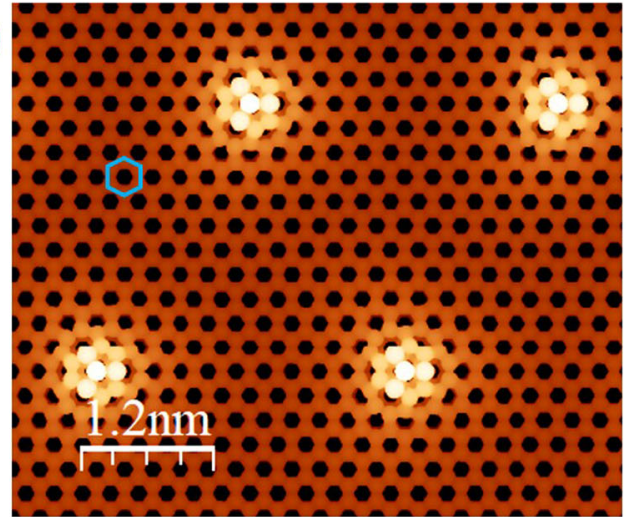

(c)

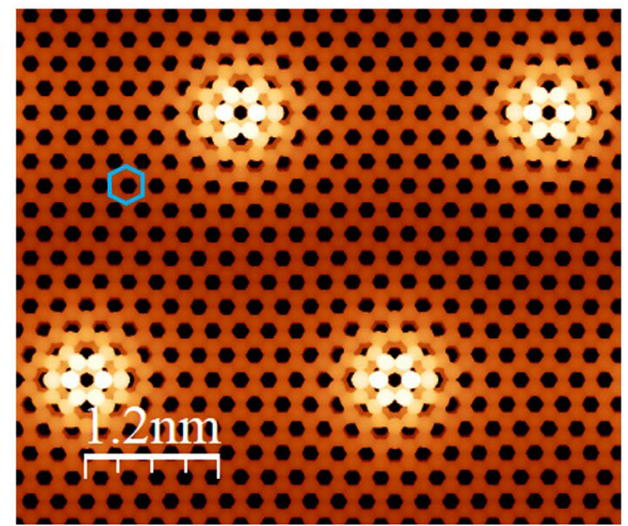

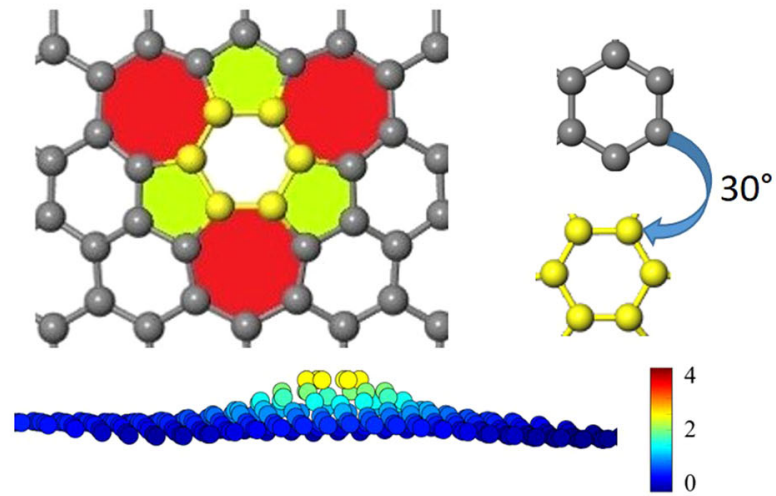

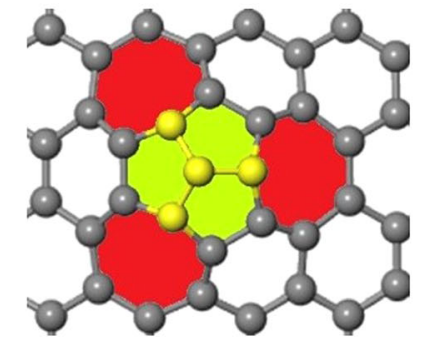

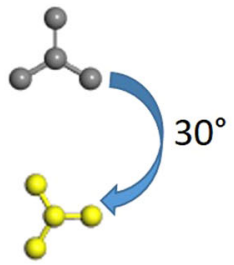

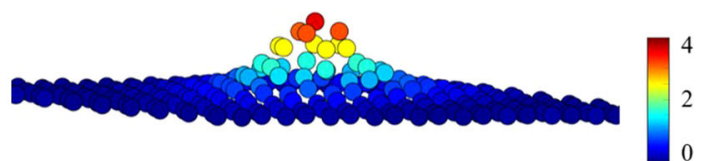

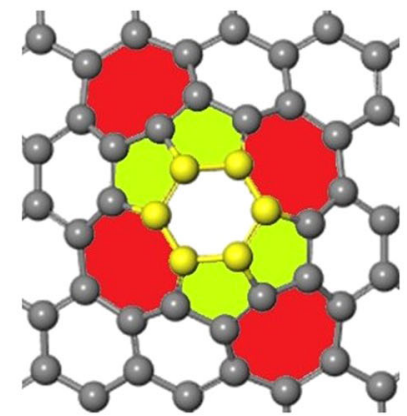

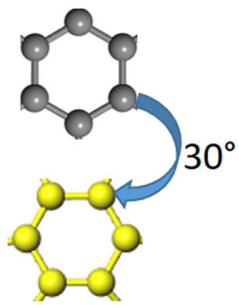

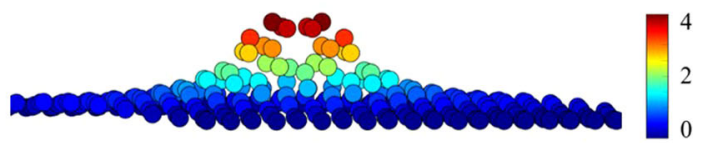

Fig. 1 The detailed structure of atomic-scale graphene blisters after structure relaxation. The simulated STM images are on the left panel, the top and side view on the right panel $\mathbf{a} \mathrm{C}_{2}, \mathbf{b} \mathrm{C}_{4}$, and $\mathbf{c} \mathrm{C}_{6}$, the red and green areas represent heptagonal and pentagonal rings, the color bars with the side view are represent out of plan distortions in $\AA$.

\section{RESULTS AND DISCUSSION}

Blister systems

Individual carbon ad-atoms on graphene are predicted to be mobile at room temperature, and thus, not expected to be found after deposition; upon encountering another carbon ad-atom, highly stable self-interstitial dimers can be formed $24,27,29$. Contrary to the vacancy defects with deficient carbon atoms, these blister structures are usually seen as defects containing up to three pairs of additional carbon atoms. Specific blisters incorporated into the graphene lattice considered in our study have been realized experimentally via $80 \mathrm{kV}$ electron beam irradiation and imaged using atomic-resolution aberration-corrected high-resolution transmission electron microscopy ${ }^{31}$. Similar blisters have also been observed in numerous other experimental studies ${ }^{24,33-35}$.
Figure 1a-c shows the different blisters and simulated STM images to match the experimental data. The notation $C_{x}$ is used to denote a blister defect containing $x$ additional carbon atoms, which are $\mathrm{C}_{2}, \mathrm{C}_{4}$, and $\mathrm{C}_{6}$ in this study. These blisters have a quasi-spherical shape with out-of-plane distortions that coexist with small wrinkling. The blister-oriented distortion increases with size and reaches its maximum value for the $C_{6}$ case. The strain induced by the out-of-plane displacement gives rise to a potential imbalance in the graphene sublattice and creates a PMF around the blister $^{18,21}$. It should be noted that the central hexagon in the $\mathrm{C}_{2}$ blister region is rotated by $30^{\circ}$ with respect to the graphene sheet and the local symmetry is modified, as shown in Fig. 1a. The modification of local symmetries can also be seen in the other two cases. The local symmetry change and the atomic buckling induced strain create localized states around the Fermi level with a 

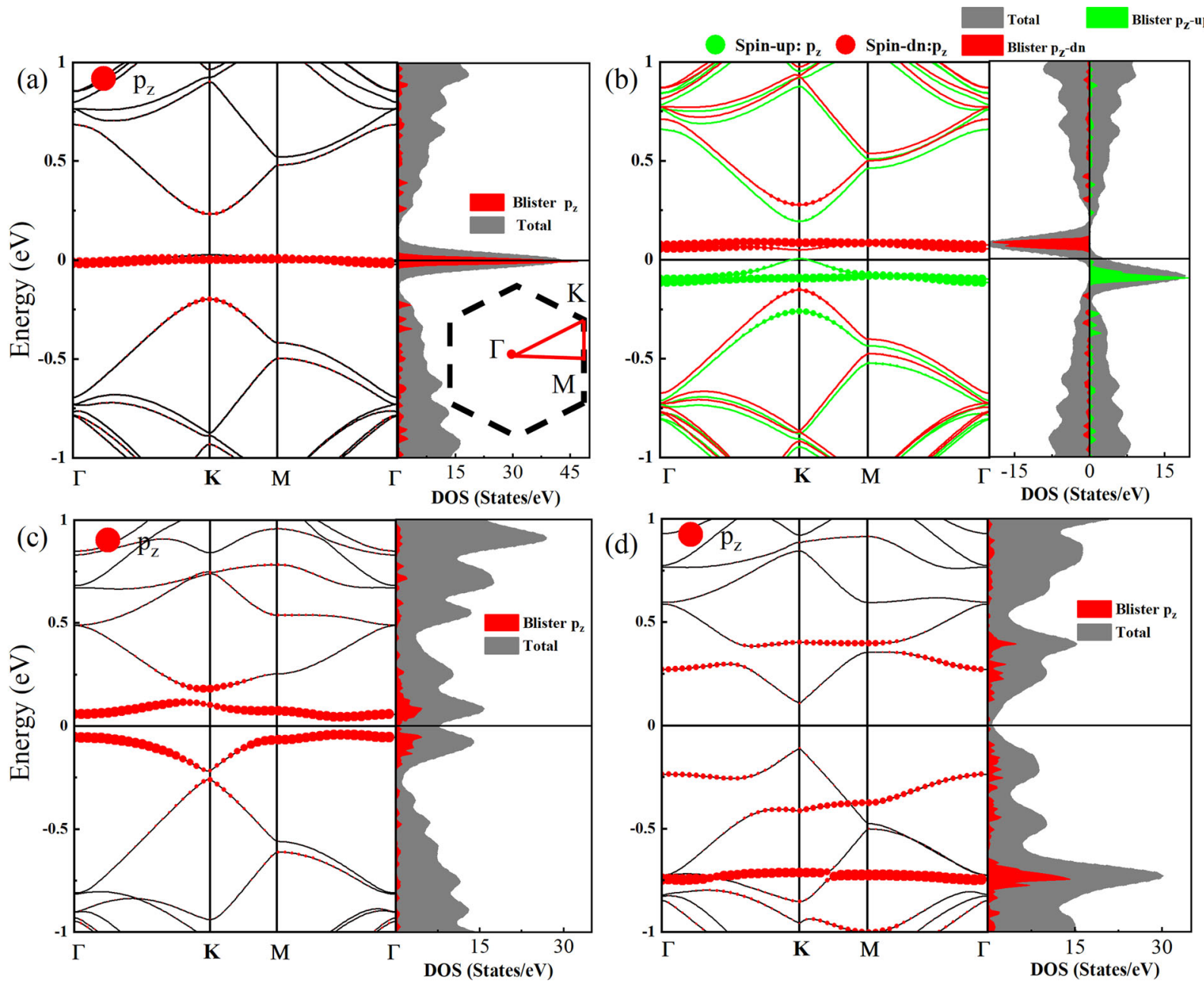

Fig. 2 Projected band structures and corresponding DOS showing Van Hove singularities. a $C_{2}$ nonmagnetic case, the red color representing the contribution from the $p_{z}$ orbitals of the blister atoms; $\mathbf{b}$ spin-polarized case of $C_{2}$, the green and red color representing the up and down spin; $\mathbf{c}, \mathbf{d}$ are for the ground state of $C_{4}$ and $C_{6}$, respectively; The size of the circles represents the relative weights of the $p_{z}$ orbitals of the blister atoms.

character that differs from pristine graphene. Here it should be noted that we have calculated all of the three blister systems in $(7 \times 7)$ and $(11 \times 11)$ supercells. The blister density in the larger supercell is $1.34 \times 10^{5} \mu \mathrm{m}^{-2}$, which is comparable to the experimental value of $(1 \pm 0.2) \times 10^{5}$ for a similar system ${ }^{36}$. We also have performed molecular dynamics (MD) simulations (at $300 \mathrm{~K}$ ) to check the thermal stability. The MD simulation results have been shown in Supplementary Fig. 1. It can be seen that the blister structures display increased out-of-plane rippling, nonetheless, the graphene sheet and blister structure are well intact at room temperature.

\section{Magnetic state}

In pristine graphene, the $\mathrm{sp}^{2}$ hybridized states consist of low energy $\sigma$-bands while the Dirac-cone is dominated by the out-ofplane $\pi$-orbital $\left(p_{\mathrm{z}}\right)$ around the Fermi level. However, localized states involving $\pi$-orbitals $\left(p_{z}\right)$ caused by the change in local symmetry and geometric distortions lead to VHS in these blister structures. In addition, the related rippling in graphene also induces a certain energy gap. Figure $2 a$ shows the band structures and density of states with projections of the $\pi$-orbitals $\left(p_{z}\right)$ of the blister atoms. In the $C_{2}$ system, the Fermi energy nearly aligns with the $2 \mathrm{D}$ VHS at $\Gamma$, where the band dispersion has a saddle point causing the density of states to diverge logarithmically. Thus, double occupation of the $\pi$-state electrons with different spins is forbidden because of electrostatic Coulomb repulsion, and the VHS splits into two spin-polarized states and results in the emergence of a magnetic state, as shown in Fig. $2 \mathrm{~b}$. The relatively smaller out of plane distortion maintains the flat bands and related VHS close to the Fermi level in $C_{2}$ (see Fig. 1a). The magnetic state due to the VHS is sensitive to the Fermi level position and can be affected by charge transfer, which usually occurs in the presence of substrates. Therefore, the influence of charge doping on the magnetic state was analyzed (see Supplementary Fig. 2). It is found that the magnetic state is stable within a doping range of $1.0 \mathrm{e}(\mathrm{h})$ per unit. The survival of the magnetic state over a large range of external charge carrier shows the robustness of the magnetic state in $C_{2}$. The presence of the blister-induced VHS is not limited to the $C_{2}$ but also observed in other blisters. In the $C_{4}$ system, the localized $\pi$ states are lowered in energy due to the increased out-of-plane distortion. Thus, the flat bands and related VHS shift away from the Fermi level (Fig. 2c), and no net magnetism is observed in this structure. However, the close proximity of the VHS to the Fermi level suggests the possibility of a magnetic state in the presence of charge doping. The alignment of the VHS with the Fermi level leads to a magnetic state in $C_{4}$, and a maximum magnetic moment value of $0.9 \mu_{B}$ is obtained with a charge doping of $1.0 \mathrm{e}$ (h) per unit (Supplementary Fig. 2). With a further increase of the carbon dimer in $\mathrm{C}_{6}$, the VHS are pushed further away from the 

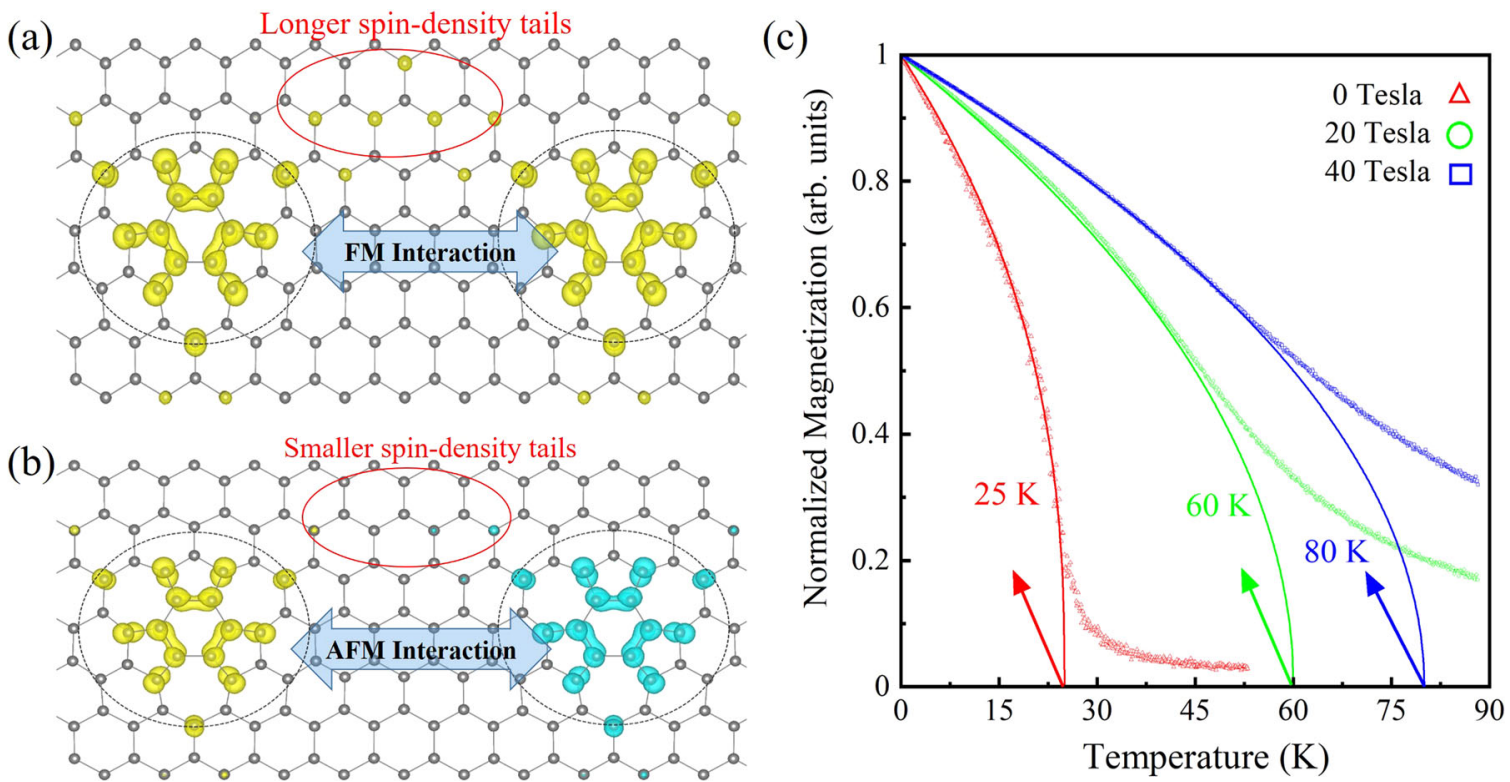

Fig. 3 Long-range ordering and magnetization density for $\mathbf{C}_{2}$. The $\mathbf{a}$ FM and $\mathbf{b}$ AFM spin configurations, with iso-surface values of \pm 0.005 e Bohr ${ }^{-3}$, the gold, and cyan colors represent the up and down spin, c MC calculations of mean magnetization as a function of temperature.

Table 1. The magnetic properties of $C_{2}$.

\begin{tabular}{lllll}
\hline Distance & NM & FM & AFM & $\mu_{B}$ \\
\hline$\sim 1$ & 138 & 0 & 26.6 & 3.70 \\
$\sim 2$ & 143 & 0 & 14.5 & 3.62 \\
\hline
\end{tabular}

Blister-to-blister distance $(\mathrm{nm})$, total energy difference $(\mathrm{meV})$ for different magnetic configurations, and magnetic moment $\left(\mu_{B}\right)$ for long-range magnetic interaction in the $C_{2}$ Blister system.

Fermi level because of the increased out-of-plane distortion (Fig. 2d). The markedly lower energies of the VHS in $\mathrm{C}_{6}$ diminish any possibility of it being in a magnetic state, even by means of charge doping. It is worthwhile to note that magnetism in graphene can also be induced by breaking the $\mathrm{sp}^{2}$ hybridization as in the hydrogen adsorption case ${ }^{37}$ or through creating zigzag edges in the ribbon case ${ }^{38}$. However, in the blistered graphene sheet, the addition of an even a number of carbon atoms $(2,4,6)$ still keeps three bonds for each carbon atom, and the typical $\mathrm{sp}^{2}$ hybridization is preserved even for the blister carbon atoms.

In the $\mathrm{C}_{2}$ structure, the VHS and related magnetic state are spread over the whole heptagonal and pentagonal ring pattern, which opens up the possibility of different short-range magnetic orderings. To check these orderings, the nonmagnetic (NM), ferromagnetic (FM), and several anti-FM (AFM) spin configurations are considered (see Supplementary Fig. 3 for details). It is found that the AFM states with all possible spin alignments always turn into FM or NM states. The absence of AFM removes the possibility of any short-range antiparallel magnetic domains in this structure. In contrast with the magnetic exchange interactions associated with strongly localized $d$-orbitals, the interactions related to the $p$ state are predicted to extend over several nanometers because of their longer wave function tails. To check the long-range magnetic ordering, the blister-to-blister magnetic coupling is investigated with distances of $\sim 1$ and $2 \mathrm{~nm}$. The local spin densities of $C_{2}$ for inter-blister FM and AFM ordering are shown in Fig. $3 a, b$, respectively, and the energetics for different magnetic states are shown in Table 1. Our results show strong FM coupling even with a blister-to-blister distance of $2 \mathrm{~nm}$. As can be seen in Fig. 3, the spin densities in the FM state have longer decaying tails compared with the AFM state. This suggests comparative delocalization of electrons, and thus, effective lowering of the kinetic energies in the FM configuration. This lowering of the kinetic energy shifts the balance in favor of the FM states. Compared with the magnetic state in graphene caused by hydrogen adsorption, where the FM state depends on distance and the sublattice adsorption site $^{37}$, the blistered graphene can have a robust FM state which is not dependent on the relative orientations of blisters. Under practical conditions, thermal fluctuations can hinder long-range magnetic ordering, hence, Monte Carlo $(\mathrm{MC})$ simulations are performed to calculate the Curie temperatures $\left(T_{C}\right)$. In the short distance regime of $\sim 1 \mathrm{~nm}$ between the two blisters, the value of $T_{\mathrm{C}}$ was $\sim 50 \mathrm{~K}$ but it sharply decreased to $25 \mathrm{~K}$ when the distance between two blisters was $\sim 2 \mathrm{~nm}$. The calculated mean magnetization as a function of temperature for a $2 \mathrm{~nm}$ distance is shown in Fig. 3c. Considering the Mermin-Wagner theorem, the low $T_{C}$ is not unexpected as thermal fluctuations can destroy the magnetic ordering in the $2 \mathrm{D}$ system. However, these thermal fluctuations can be counteracted via an external magnetic field. From Fig. $3 c$, it can be seen that the $T_{\mathrm{C}}$ can be enhanced to $80 \mathrm{~K}$ in the presence of a small external magnetic field of $40 \mathrm{~T}$ in our MC simulations.

\section{Valley Hall effect}

To describe the symmetry-related character of the Bloch electron, we calculate the Berry curvature $\Omega(\boldsymbol{k})$, which can be obtained from the overlap matrix of the Kohn-Sham orbitals, as $\Omega_{\mathrm{n}}(\boldsymbol{k})=\nabla \times \mathbf{A}_{n \boldsymbol{k}}$, where $\mathbf{A}_{n \mathbf{k}}=-i\left\langle u_{n \boldsymbol{k}}\left|\frac{\partial}{\partial \boldsymbol{k}}\right| u_{n \boldsymbol{k}}\right\rangle$ and $\left|\mu_{n k}\right\rangle$ is a normalized wave function. The $k$-resolved Berry curvature $\Omega(\boldsymbol{k})$ integrated over the whole valence band of $C_{2}$ is shown in Fig. 4a. Externally introduced exchange splitting without lattice deformation results in $\Omega(\boldsymbol{k})=\Omega(-\boldsymbol{k})$ because of the preservation of inversion symmetry ${ }^{3,39,40}$. In contrast, the Berry curvature observed here has two opposite peaks at the BZ corners $\left(K\right.$ and $\left.K^{\prime}\right)$ in $C_{2}$. This can be attributed to the remarkable relationship of Dirac fermions to sublattice polarization and inversion symmetry breaking caused by mechanical deformation. Blister induced deformation and local symmetry modification cause a pronounced PMF, even in the vicinity far beyond the blister region because of long-range rippling. Similar phenomena have been predicted in previous theoretical works ${ }^{41,42}$. Here the $C_{2}$ system loses its spatial inversion, but the local structure and bands related to the VHS 
(a)

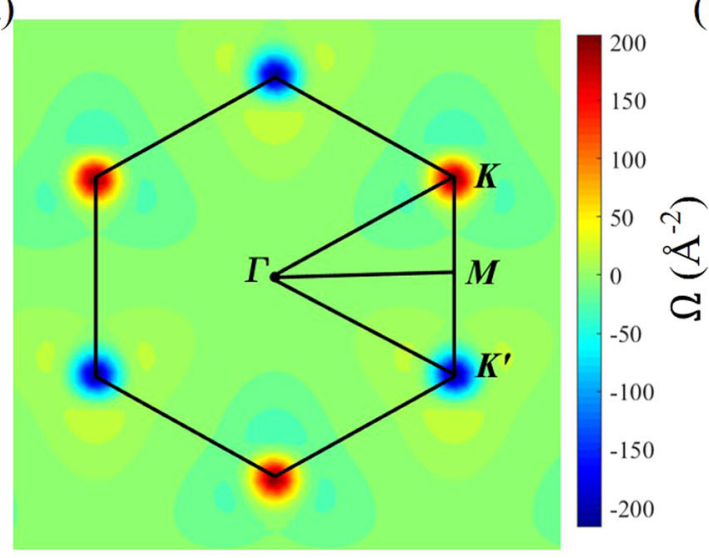

(c)

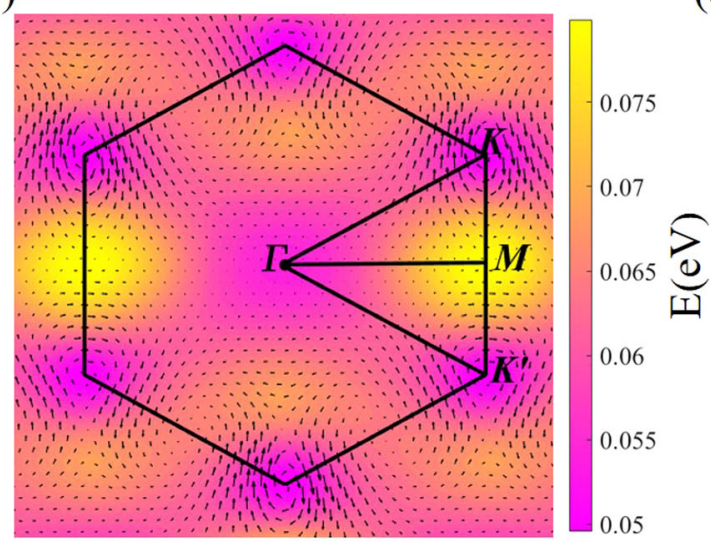

(b)

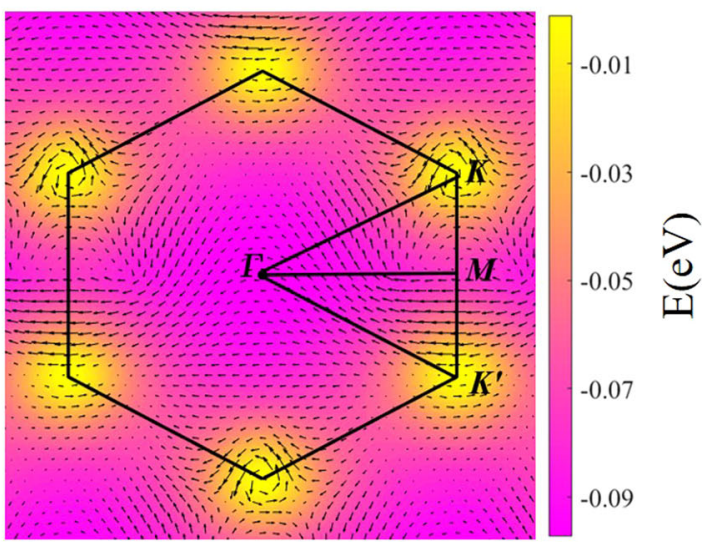

(d)

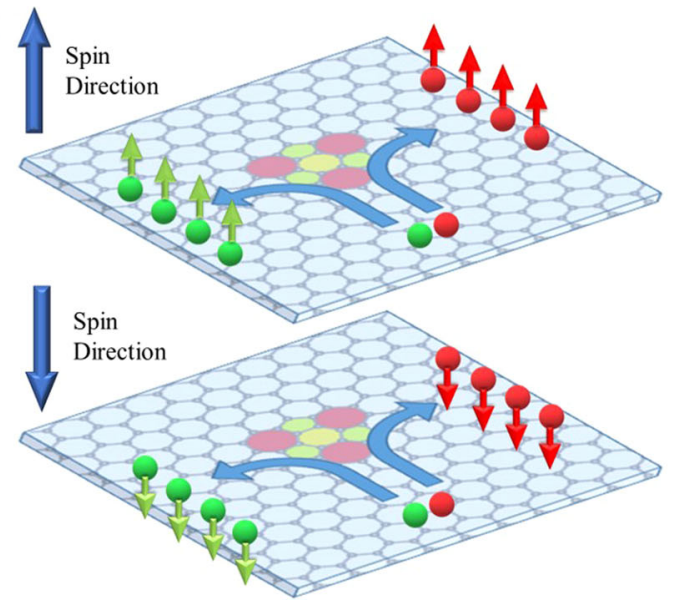

Fig. 4 The nature of Hall conductivity in $C_{2}$. a The momentum resolved Berry curvature for the occupied bands. The first BZ is denoted by the black hexagon, and the color code is in units of $\left(\AA^{-2}\right)$. In-plane spin textures for VBM (b) and CBM (c). The color scale refers to the band energy $(\mathrm{eV})$ with respect to the Fermi level, and the relative strength of the in-plane spin field $S_{x y}$ is represented by black arrows. $\mathbf{d}$ Schematic of the spin filtered VH effects.

have preserved threefold symmetry. The reduction of sixfold to threefold symmetry creates equal and opposite distinguishable chiral valleys at $K$ and $K^{\prime}$. It should be noted that the absence of spatial and time inversion symmetry should provoke a valley polarization state. However, due to the insignificant SOC strength of carbon atoms, no tangible valley polarization is observed in $\Omega$ $(\boldsymbol{k})$. The out-of-plane magnetization shows no substantial change in the $\boldsymbol{S}_{z}$ spin component throughout the BZ (not shown here). Nonetheless, the in-plane spin textures $\left(\mathbf{S}_{x y}\right)$ for both the valence band maxima (VBM) and conduction band minima (CBM) show chirality, as shown in Fig. 4b, c. The VBM has a clockwise and counter-clockwise spin texture around $K\left(K^{\prime}\right)$, which matches well with the threefold symmetry of the Berry curvature at $K\left(K^{\prime}\right)$. Furthermore, the valley polarization is quite visible in the $S_{x y}$ vector field of the VBM and CBM because the in-plane spin field is different at $K$ compared with $K^{\prime}$. The anomalous Hall conductivity (AHC) can be defined as $\sigma_{x y}^{A H C}=\frac{\mathrm{e}^{2}}{2 \pi h} \int_{B Z} \Omega(\mathbf{k}) \mathbf{d} \mathbf{k}_{\mathbf{x}} \mathbf{d} \mathbf{k}_{\mathbf{y}}$, where $k_{x}$ and $k_{y}$ are BZ vectors. Therefore, in the absence of valley polarization $\Omega$ $(\mathbf{k})=-\Omega(-\mathbf{k})$, and the total AHC $\sigma_{x y}^{\mathrm{AHC}}=\sigma_{x y}^{K}+\sigma_{x y}^{K}$ is zero. The socalled valley Hall conductivity can be defined as $\sigma_{x y}^{\mathrm{VHC}}=\frac{\mathrm{e}^{2}}{2 \pi \mathrm{h}} \int_{\mathrm{k} / k^{\prime}} \Omega(\mathbf{k}) \mathrm{d} \mathbf{k}_{\mathrm{x}} \mathrm{d} \mathbf{k}_{\mathrm{y}}$ at the two valley points $K\left(K^{\prime}\right)$. Because of exchange splitting, we obtain a value of $1 / 2(-1 / 2)$ in the unit of $\frac{e^{2}}{h}$, which confirms VHE in $C_{2}$. Consequently, the electrons from both valleys are deflected to opposite edges, and the contributions to the AHC from the individual valleys $\sigma_{x y}^{A H C} \neq 0$. A similar case was also discussed previously with the Fermi-level locating in the band gap ${ }^{43}$. The spin polarization in $C_{2}$ gives rise to large exchange splitting and spin-filtered $\mathrm{VH}$ states can be observed, where all the current-carrying edge states have the same spin orientation. Thus, this $C_{2}$ blister system also presents the spinfiltered Hall Effect, as shown in Fig. 4d. Moreover, the spin-filtered $\mathrm{VH}$ states are protected against the slight movement of the Fermi level caused by exchange splitting. To extract the graphene character in $\mathrm{C}_{2}$, we also calculate the Berry curvatures by integrating over the graphene valence bands (impurity bands near the Fermi level are excluded). In contrast with pristine graphene, the Berry curvatures of the valence bands without impurity bands also show a chiral character $\Omega(K)=-\Omega\left(K^{\prime}\right)$ (see Supplementary Fig. 4), which makes it a robust candidate for practical valleytronics devices.

The band structures in Fig. 2 have been presented along the high symmetry lines. However, under strain, the Dirac point shifts away from the chosen high symmetry path. To clarify this shift in $\mathrm{C}_{4}$, we present the $3 \mathrm{D}$ band plot $\mathrm{E}\left(k_{x}, k_{y}\right)$ in Fig. $5 \mathrm{a}$. The $\mathrm{C}_{4}$ system has the opposite Berry curvature spikes shifted from the BZ points $K / K^{\prime}$ to points $L / L^{\prime}$, as shown in Fig. $5 b$. The Berry curvature peaks around $\Gamma$ correspond to the avoided band crossing points between the VBM and CBM, as can be seen in the 3D band. This shift from the actual $K$ to $L$ points is due to local symmetry change, and this phenomenon has also been observed in strained graphene ${ }^{44}$. Furthermore, the inversion symmetry breaking induced by lattice deformation generates Rashba splitting, which can be seen in the spin texture of the VBM and CBM, as shown in Fig. $5 c$, d, respectively. The in-plane spin texture $\mathbf{S}_{\mathbf{x y}}$ presents a helical feature in the vicinity of the BZ center. Similar to $C_{4}$, the opposite Berry curvature peaks in the $C_{6}$ shift from the $K\left(K^{\prime}\right)$ point 

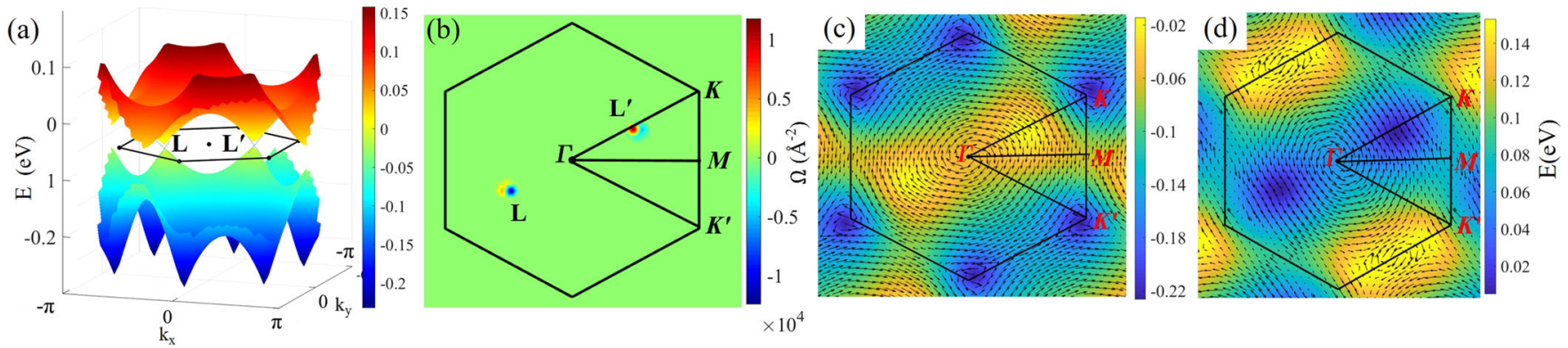

(e)
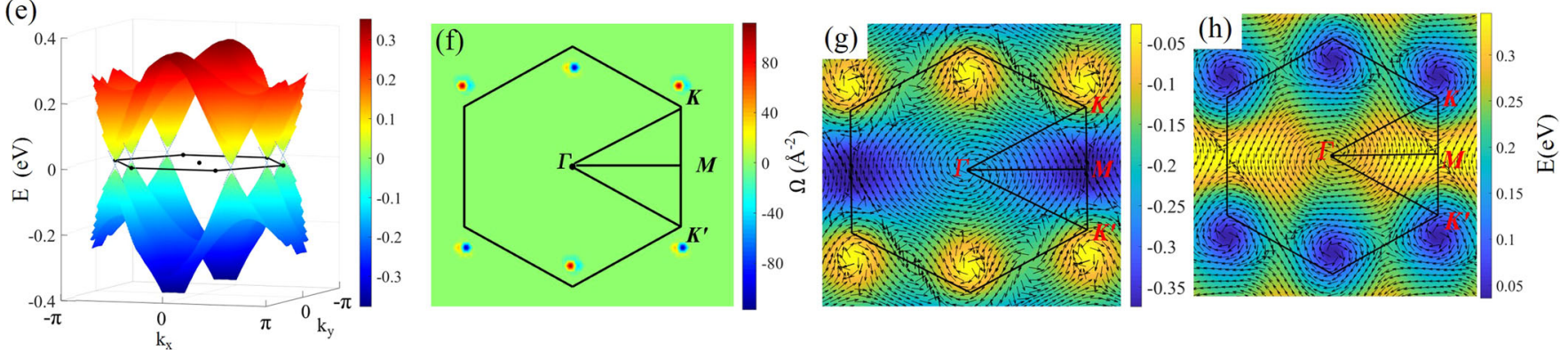

Fig. 5 The Hall conductivity nature of $\mathbf{C}_{\mathbf{4}}$ and $\mathbf{C}_{\mathbf{6}}$ systems. Upper panel for $C_{4}$ structure: $\mathbf{a}$ The $3 D$ view of the band dispersions; $\mathbf{b}$ the momentum resolved Berry curvature for the occupied bands, where the color code is in the unit $\left(\AA^{-2}\right)$; $\mathbf{c}$, $\mathbf{d}$ are the spin textures for the VBM and $\mathrm{CBM}$, respectively. The color scale refers to the band energy $(\mathrm{eV})$ with respect to the Fermi level and the black arrows represent the relative strength of the in-plane spin field $S_{x y}$. The lower panel is the same as the upper panel for $C_{6}$ structure.

with the shift of the Dirac points (Fig. 5e, f). This is because the four pairs of heptagonal and pentagonal rings induce strain, which further reduces the local symmetry from three to twofold. Figure $5 \mathrm{~g}$, h demonstrates the existence of in-plane chiral spin texture around the displaced Dirac points. The CBM spin texture differs from that of the VBM, nonetheless, it preserves the helical spin texture with different chirality. The absence of exchange splitting in $C_{4}$ and $C_{6}$ means the preservation of time-reversal symmetry and the opposite Berry curvature of the two valleys induced by the PMF does not give rise to any $\mathrm{AHC}\left(\sigma_{x y}^{\mathrm{AHC}}=0\right)$. However, even though the overall time-reversal symmetry is preserved, the PMF generates VHE with the opposite sign of $\sigma_{x y}^{K / K^{\prime}}$ in the two valleys. The $\sigma_{x y}^{\mathrm{VHC}}= \pm 1$ in the presence of very weak SOC because the two spin states are degenerate in both valleys. Such VHE causes the splitting of the current into two different valleys that originally move in the same direction. In comparison with ordinary semiconductors, the robust chiral spin texture at the Dirac cones should markedly suppress the back-scattering of charge carriers. It should be noted that the results can be affected by the strong interaction with a substrate. However, it is anticipated that these properties will be preserved in the case of an insulating or wide gap semiconductor substrate.

In conclusion, we investigate the AHE and VHE in the blistered graphene sheets. The blister structures are composed of pentagonal and heptagonal rings of carbon atoms, which show a strong tendency toward out-of-plane deformation with increasing blister size. The geometric distortion leads to VHS which could induce exchange splitting in otherwise paramagnetic graphene. Unlike the ribbon edges, these atomic-scale blisters are fully immersed within the graphene sheet, hence, its magnetic state is protected from contamination and reconstruction effects that could hamper experimental detection. We have shown that in the presence of exchange splitting, the graphene blister hosts the spin-filtered VHE in units of half $\pm \frac{\mathrm{e}^{2}}{h}$. We propose that the distinguishable chiral valley pseudospin state $(\Omega(\mathrm{K}) \sim-\Omega(-\mathrm{K}))$ can occur even with magnetism. In the presence of time inversion, the graphene blister shows VHE with the opposite sign of $\sigma_{x y}^{K / K \prime}$ in the two valleys with the unit of $\pm \frac{\mathrm{e}^{2}}{h}$. Our study provides a pathway to realize the spin-filtered and valley contrasting VH effects in the absence of external fields in graphene.

\section{METHODS}

Numerical methods

The first-principles calculations are mainly performed by using OpenMX code, which is based on a linear combination of pseudoatomic orbital formalism ${ }^{45,46}$. Pseudo-atomic basis functions of $s^{2} p^{2} d^{1}$ along with the norm-conserving pseudopotentials are used from the OpenMX library. The electronic exchange-correlation interactions are treated in the generalized gradient approximation (GGA) of Perdew-Burke-Ernzerhof ${ }^{47}$. To avoid the interactions between images created by the periodic boundary condition, a vacuum space of $15 \AA$ is used in the nonperiodic direction. To model the blisters the primitive vectors and atomic positions are fully relaxed with the convergence criterion of $0.01 \mathrm{eV} \AA^{-1}$ for interatomic forces. The energy cut-off of $200 \mathrm{Ry}$ is used and the energy convergence criterion is kept $2 \times$ $10^{-6} \mathrm{eV}$ for each electronic structure calculation. We have performed the calculations by using two supercell sizes of $7 \times 7$ and $11 \times 11$ to confirm that the graphene sheets with different blister densities indeed show similar electronic properties. Brillouin zone is sampled by $(7 \times 7 \times 1)$ and $(5 \times 5 \times 1) \Gamma$-centered $\mathrm{k}$-mesh for supercell size of $7 \times 7$ and $11 \times 11$, respectively. The scanning tunneling microscopy (STM) images are simulated by using the Tersoff-Hamann theory for STM images ${ }^{48}$, as supplied in the OpenMX code and WSXM software is used for presentation ${ }^{49}$. We also double-checked the electronic and magnetic state of blisters with the Vienna ab initio simulation package ${ }^{50,51}$ at the level of the spin-polarized $\mathrm{GGA}^{47}$. The interaction between valence electrons and ionic cores was described within the framework of the projector augmented wave method ${ }^{52}$. The energy cutoff for the plane-wave basis expansion was set to $500 \mathrm{eV}$.

\section{Spin texture and berry curvature}

The spinor wave function $\psi_{\sigma}(\mathbf{r}, \mathbf{k}, \boldsymbol{\mu})$ obtained from the non-collinear firstprinciples calculations are used to obtain the spin density matrix $\mathrm{P}_{\sigma \sigma^{\prime}}(\mathbf{k}, \mu)$ by using

$\mathrm{P}_{\sigma \sigma \prime}(\mathbf{k}, \mu)=\int \psi_{\sigma}^{*}(\mathbf{r}, \mathbf{k}, \mu) \psi_{\sigma \prime}(\mathbf{r}, \mathbf{k}, \mu) d \mathbf{r}$,

where $\mu$ and $\mathbf{k}$ are the band index and reciprocal space points respectively, $\sigma$ and $\sigma^{\prime}$ are two opposite spin indices $\left(\uparrow\right.$ or $\downarrow$ ). The wave function $\psi_{\sigma}(\mathbf{r}, \mathbf{k}, \boldsymbol{\mu})$ 
in OpenMX is just a linear combination of pseudo atomic orbitals. The spin polarization $\left(P_{x}, P_{y}, P_{z}\right)$ is deduced in the k-space from the $2 \times 2$ spin density matrix ${ }^{53}$. Berry curvature is calculated by using the Fukui-Hatsugai-Suzuki method $^{54}$. In this method, the overlap matrix $U$ is defined by ${ }^{55}$

$\mathrm{U}_{\Delta \mathrm{k}}(\mathbf{k})=\operatorname{det}\left\langle\mathrm{u}_{\mathrm{n}}(\mathbf{k}) \mid \mathrm{u}_{\mathrm{m}}(\mathbf{k}+\Delta \mathbf{k})\right\rangle$,

and Berry curvature is defined by

$\mathrm{F}(\mathbf{k})=\operatorname{Im} \log \mathrm{U}_{\Delta \mathrm{k}_{1}}(\mathbf{k}) \mathrm{U}_{\Delta \mathrm{k}_{2}}\left(\mathbf{k}+\Delta \mathbf{k}_{1}\right) \mathrm{U}_{\Delta \mathrm{k}_{1}}^{-1}\left(\mathbf{k}+\Delta \mathbf{k}_{2}\right) \mathrm{U}_{\Delta \mathrm{k}_{2}}^{-1}(\mathbf{k})$.

\section{Spin dynamics}

Curie temperature $\left(T_{C}\right)$ is a critical point at which the FM system becomes paramagnetic, and it is an important parameter to evaluate magnetic properties. We simulate the temperature-dependent magnetization curve and extracted the $T_{\mathrm{C}}$ by carrying out $\mathrm{MC}$ simulations ${ }^{56}$. During the MC simulations, $45 \times 45$ supercells is used to mimic the $2 \mathrm{D}$ lattice; this is found to be large enough to minimize the periodic constraints. The MC simulations perform a stepwise loop overtemperature, performing equilibration and averaging steps and calculating the mean magnetization before incrementing the temperature. The number of equilibration and averaging steps increased stepwise and the optimized value is found to be 30,000 . The temperature-dependent magnetization curve is fitted by using the Curie-Bloch equation in the classical limit as given below

$m(T)=\left[1-T / T_{\mathrm{c}}\right]^{\beta}$,

where $T$ is the temperature and $T_{\mathrm{C}}$ is the Curie temperature.

The interactions between the blister and external applied field $\left(H_{\text {ext }}\right)^{56}$ is defined by

$H_{\text {ext }}=-\sum_{i} \mu_{s} S_{i} \cdot H_{\text {ext }}$

where $\mu_{s}$ is the atomic magnetic moment and $S_{i}$ is a unit vector denoting the local spin moment direction, respectively.

\section{DATA AVAILABILITY}

The data that support the findings of this study are available upon request to the corresponding author.

\section{CODE AVAILABILITY}

The central code used in this study is Openmx. Further information regarding licensing and code documentation can be found at http://www.openmx-square.org.

Received: 10 July 2020; Accepted: 4 November 2020;

Published online: 17 December 2020

\section{REFERENCES}

1. $\mathrm{Yu}, \mathrm{R}$. et al. Quantized anomalous Hall effect in magnetic topological insulators. Science 329, 61-64 (2010).

2. Zhang, F., Jung, J., Fiete, G. A., Niu, Q. \& MacDonald, A. H. Spontaneous quantum Hall states in chirally stacked few-layer graphene systems. Phys. Rev. Lett. 106, 156801 (2011).

3. Qiao, Z. et al. Quantum anomalous Hall effect in graphene from Rashba and exchange effects. Phys. Rev. B 82, 161414 (2010)

4. Wang, Z., Tang, C., Sachs, R., Barlas, Y. \& Shi, J. Proximity-induced ferromagnetism in graphene revealed by the anomalous Hall effect. Phys. Rev. Lett. 114, 016603 (2015).

5. Leutenantsmeyer, J. C., Kaverzin, A. A., Wojtaszek, M. \& Wees, B. Jvan Proximity induced room temperature ferromagnetism in graphene probed with spin currents. 2D Mater. 4, 014001 (2016).

6. Hallal, A., Ibrahim, F., Yang, H., Roche, S. \& Chshiev, M. Tailoring magnetic insulator proximity effects in graphene: first-principles calculations. 2D Mater. 4, 025074 (2017).

7. Thouless, D. J., Kohmoto, M., Nightingale, M. P. \& den Nijs, M. Quantized Hall conductance in a two-dimensional periodic potential. Phys. Rev. Lett. 49, 405-408 (1982).

8. Cao, T. et al. Valley-selective circular dichroism of monolayer molybdenum disulphide. Nat. Commun. 3, 1-5 (2012).
9. Mak, K. F., He, K., Shan, J. \& Heinz, T. F. Control of valley polarization in monolayer MoS 2 by optical helicity. Nat. Nanotechnol. 7, 494-498 (2012).

10. Pesin, D. \& MacDonald, A. H. Spintronics and pseudospintronics in graphene and topological insulators. Nat. Mater. 11, 409-416 (2012).

11. Xiao, D., Liu, G.-B., Feng, W., Xu, X. \& Yao, W. Coupled spin and valley physics in monolayers of $\mathrm{MoS}_{2}$ and other group-VI dichalcogenides. Phys. Rev. Lett. 108 196802 (2012).

12. Schaibley, J. R. et al. Valleytronics in 2D materials. Nat. Rev. Mater. 1, 1-15 (2016)

13. Castro Neto, A. H., Guinea, F., Peres, N. M. R., Novoselov, K. S. \& Geim, A. K. The electronic properties of graphene. Rev. Mod. Phys. 81, 109-162 (2009).

14. Abergel, D. S. L., Apalkov, V., Berashevich, J., Ziegler, K. \& Chakraborty, T. Properties of graphene: a theoretical perspective. Adv. Phys. 59, 261-482 (2010).

15. Guinea, F., Katsnelson, M. I. \& Geim, A. K. Energy gaps and a zero-field quantum Hall effect in graphene by strain engineering. Nat. Phys. 6, 30-33 (2010).

16. de Juan, F., Cortijo, A., Vozmediano, M. A. H. \& Cano, A. Aharonov-Bohm interferences from local deformations in graphene. Nat. Phys. 7, 810-815 (2011).

17. Guinea, F., Geim, A. K., Katsnelson, M. I. \& Novoselov, K. S. Generating quantizing pseudomagnetic fields by bending graphene ribbons. Phys. Rev. B 81, 035408 (2010).

18. Levy, N. et al. Strain-induced pseudo-magnetic fields greater than 300 tesla in graphene nanobubbles. Science 329, 544-547 (2010).

19. Li, S.-Y. et al. Observation of unconventional splitting of Landau levels in strained graphene. Phys. Rev. B 92, 245302 (2015).

20. Venderbos, J. W. F. \& Fu, L. Interacting Dirac fermions under a spatially alternating pseudomagnetic field: realization of spontaneous quantum Hall effect. Phys. Rev. B 93, 195126 (2016).

21. Georgi, A. et al. Tuning the pseudospin polarization of graphene by a pseudomagnetic field. Nano Lett. 17, 2240-2245 (2017).

22. Klimov, N. N. et al. Electromechanical properties of graphene drumheads. Science 336, 1557-1561 (2012)

23. Banhart, F., Kotakoski, J. \& Krasheninnikov, A. V. Structural defects in graphene. ACS Nano 5, 26-41 (2011).

24. Lehtinen, O., Vats, N., Algara-Siller, G., Knyrim, P. \& Kaiser, U. Implantation and atomicscale investigation of self-interstitials in graphene. Nano Lett. 15, 235-241 (2015).

25. Bunch, J. S. et al. Impermeable atomic membranes from graphene sheets. Nano Lett. 8, 2458-2462 (2008)

26. Sun, L., Banhart, F. \& Warner, J. Two-dimensional materials under electron irradiation. MRS Bull. 40, 29-37 (2015).

27. Gong, C. et al. Thermally induced dynamics of dislocations in graphene at atomic resolution. ACS Nano 9, 10066-10075 (2015).

28. Zhang, K. \& Arroyo, M. Coexistence of wrinkles and blisters in supported graphene. Extrem. Mech. Lett. 14, 23-30 (2017).

29. Lusk, M. T. \& Carr, L. D. Nanoengineering defect structures on graphene. Phys. Rev. Lett. 100, 175503 (2008).

30. Li, G. et al. Observation of Van Hove singularities in twisted graphene layers. Nat. Phys. 6, 109-113 (2010)

31. Robertson, A. W., He, K., Kirkland, A. I. \& Warner, J. H. Inflating graphene with atomic scale blisters. Nano Lett. 14, 908-914 (2014).

32. Li, S.-Y., Su, Y., Ren, Y.-N. \& He, L. Valley polarization and inversion in strained graphene via pseudo-landau levels, valley splitting of real landau levels, and confined states. Phys. Rev. Lett. 124, 106802 (2020).

33. Meyer, J. C. et al. Direct imaging of lattice atoms and topological defects in graphene membranes. Nano Lett. 8, 3582-3586 (2008).

34. Kotakoski, J., Krasheninnikov, A. V., Kaiser, U. \& Meyer, J. C. From point defects in graphene to two-dimensional amorphous carbon. Phys. Rev. Lett. 106, 105505 (2011)

35. Kotakoski, J., Mangler, C. \& Meyer, J. C. Imaging atomic-level random walk of a point defect in graphene. Nat. Commun. 5, 1-5 (2014).

36. Lu, J., Neto, A. H. C. \& Loh, K. P. Transforming moiré blisters into geometric graphene nano-bubbles. Nat. Commun. 3, 823 (2012).

37. González-Herrero, $\mathrm{H}$. et al. Atomic-scale control of graphene magnetism by using hydrogen atoms. Science 352, 437-441 (2016).

38. Magda, G. Z. et al. Room-temperature magnetic order on zigzag edges of narrow graphene nanoribbons. Nature 514, 608-611 (2014).

39. Qiao, Z. et al. Quantum anomalous Hall effect in graphene proximity coupled to an antiferromagnetic insulator. Phys. Rev. Lett. 112, 116404 (2014).

40. Zhang, J., Zhao, B., Yao, Y. \& Yang, Z. Robust quantum anomalous Hall effect in graphene-based van der Waals heterostructures. Phys. Rev. B 92, 165418 (2015).

41. Pacheco Sanjuan, A. A., Wang, Z., Imani, H. P., Vanević, M. \& Barraza-Lopez, S Graphene's morphology and electronic properties from discrete differential geometry. Phys. Rev. B 89, 121403 (2014).

42. Settnes, M., Power, S. R. \& Jauho, A.-P. Pseudomagnetic fields and triaxial strain in graphene. Phys. Rev. B 93, 035456 (2016).

43. Dyrdał, A. \& Barnaś, J. Anomalous, spin, and valley Hall effects in graphene deposited on ferromagnetic substrates. 2D Mater. 4, 034003 (2017).

44. Battilomo, R., Scopigno, N. \& Ortix, C. Berry curvature dipole in strained graphene: a Fermi surface warping effect. Phys. Rev. Lett. 123, 196403 (2019). 
45. Ozaki, T. \& Kino, H. Numerical atomic basis orbitals from H to Kr. Phys. Rev. B 69, 195113 (2004).

46. Ozaki, T. Variationally optimized atomic orbitals for large-scale electronic structures. Phys. Rev. B 67, 155108 (2003).

47. Perdew, J. P., Burke, K. \& Ernzerhof, M. Generalized gradient approximation made simple. Phys. Rev. Lett. 77, 3865-3868 (1996).

48. Tersoff, J. \& Hamann, D. R. Theory of the scanning tunneling microscope. Phys. Rev. B 31, 805-813 (1985).

49. Horcas, I. et al. WSXM: a software for scanning probe microscopy and a tool for nanotechnology. Rev. Sci. Instrum. 78, 013705 (2007).

50. Kresse, G. \& Furthmüller, J. Efficiency of ab-initio total energy calculations for metals and semiconductors using a plane-wave basis set. Comput. Mater. Sci. 6, 15-50 (1996).

51. Kresse, G. \& Furthmüller, J. Efficient iterative schemes for ab initio total-energy calculations using a plane-wave basis set. Phys. Rev. B 54, 11169-11186 (1996).

52. Blöchl, P. E. Projector augmented-wave method. Phys. Rev. B 50, 17953-17979 (1994).

53. Kotaka, H., Ishii, F. \& Saito, M. Rashba effect on the structure of the bi one-bilayer film: fully relativistic first-principles calculation. Jpn. J. Appl. Phys. 52, 035204 (2013).

54. Fukui, T., Hatsugai, Y. \& Suzuki, H. Chern numbers in discretized Brillouin zone: efficient method of computing (spin) Hall conductances. J. Phys. Soc. Jpn. 74, 1674-1677 (2005)

55. Sawahata, H., Yamaguchi, N., Kotaka, H. \& Ishii, F. First-principles study of electricfield-induced topological phase transition in one-bilayer Bi(111). Jpn. J. Appl. Phys. 57, 030309 (2018).

56. Evans, R. F. L. et al. Atomistic spin model simulations of magnetic nanomaterials. J. Phys. 26, 103202 (2014).

\section{ACKNOWLEDGEMENTS}

Work at SUSTech was supported by the National Natural Science Foundation of China under Grant no. 11774142, and the Shenzhen Basic Research Fund under Grant nos. JCYJ20170817105132549, JCYJ20180504165817769, and JCYJ20170817105201098. Computational time was supported by the Center for Computational Science and Engineering of Southern University of Science and Technology. Work in Japan was partially supported by JSPS Grants-in-Aid for Scientific Research through KAKENHI, Grant no. 19K15381. Numerical calculations were carried out using the computer facilities of the Center for Computational Sciences at the University of Tsukuba through the Multidisciplinary Cooperative Research Project-2020 (Project ID: Xg20i067) and Research Institute for Information Technology Kyushu University.

\section{AUTHOR CONTRIBUTIONS}

M.-U.F. and A.H. conceived the idea. A.H. performed the D.F.T. calculations and spin dynamics simulations. M.-U.F. described the symmetry-related quantum valley Hall effects, Berry curvatures, and spin textures. L.H. and T.O. supervised the work, verified overall results, and commented on the paper. All the authors contributed to the preparation of the paper.

\section{COMPETING INTERESTS}

The authors declare no competing interests.

\section{ADDITIONAL INFORMATION}

Supplementary information is available for this paper at https://doi.org/10.1038/ s41524-020-00470-9.

Correspondence and requests for materials should be addressed to A.H. or L.H.

Reprints and permission information is available at http://www.nature.com/ reprints

Publisher's note Springer Nature remains neutral with regard to jurisdictional claims in published maps and institutional affiliations.

(c) Open Access This article is licensed under a Creative Commons (c) Attribution 4.0 International License, which permits use, sharing, adaptation, distribution and reproduction in any medium or format, as long as you give appropriate credit to the original author(s) and the source, provide a link to the Creative Commons license, and indicate if changes were made. The images or other third party material in this article are included in the article's Creative Commons license, unless indicated otherwise in a credit line to the material. If material is not included in the article's Creative Commons license and your intended use is not permitted by statutory regulation or exceeds the permitted use, you will need to obtain permission directly from the copyright holder. To view a copy of this license, visit http://creativecommons. org/licenses/by/4.0/.

(c) The Author(s) 2020 